\title{
Displacement of Actin Filament and Actin Binding Proteins under Local Deformation Processed by Digital Image Correlation Method in a Myoblast"
}

\author{
Takeomi MIZUTANI $^{* *}$, Kenichi DOI**, Yasuyuki MORITA ${ }^{* * *}$, \\ Masakazu UCHINO $^{* * * *}$, Mitsugu TODO ${ }^{* * *}$, Hisashi HAGA ${ }^{* *}$ \\ and Kazushige KAWABATA** \\ **Division of Biological Sciences, Graduate School of Science, Hokkaido University, \\ North 10 West 8, Kita-ku, Sapporo 060-0810, JAPAN \\ E-mail: mizutani@sci.hokudai.ac.jp \\ ${ }^{* * *}$ Research Institute for Applied Mechanics, Kyushu University, \\ ****Fukuoka Industrial Technology Center,
}

\begin{abstract}
Displacement of actin filament and its binding proteins in mouse myoblasts under locally applied deformation was analyzed by manual method or digital image correlation method. Cyotoskeletal components labeled by immunofluorescent technique or green fluorescent protein-fused protein were deformed via the movement of a glass needle which was poked into a cell. First, we confirmed the digital image correlation method is able to use to analyze displacement map by comparison of the manual method. Next, we examined whether the applied deformation isotropically propagates into cell body. At focal adhesions, fluorescent signals from the deformed area unchanged under the application. Mainly, focal adhesions around the poked area were moved to the direction of the movement of the needle. In addition, some adhesions away from the poked area were moved. Similar results were observed in phalloidin-stained cells. Finally, we applied the local deformation to live cells. However, displacement at the locally deformed area was not observed due to the disappearance of fluorescent signals. These results indicate that applied deformation propagated heterogeneously into a cell, and may imply that biochemical signals disrupt actin fibers under local deformation.
\end{abstract}

Key words: Mechanical Loading, Digital Image Correlation Method, Cytoskeletal Network, Remodeling

\section{Introduction}

Mechanical loading applied to a cell play a critical role in regulating cell proliferation, growth and death (see review an article by Blain ${ }^{(1)}$ ), gene expression ${ }^{(2,3)}$. At first, the loading will change configuration of cytoskeletal networks and plasma membrane, and then, is thought to be transduced into biochemical signals. The correspondence between loading and proposed biochemical signals seems to be unclear yet. To solve the problem, both map of mechanical information and the following biochemical signals should be simultaneously measured.

In order to measure mechanical information in a cell, some techniques have been developed. Specially, measurement of strain around cytoskeletons is focused because changes in cytoskeletal networks are able to be observed by optical microscopy ${ }^{(4,5)}$. Hu et 
al. developed a technique to observe the transmission of mechanical stresses within the cytoplasm using fluorescent protein-labeled cytoskeletal protein and force via the movemtent of magnetic bead ${ }^{(6)}$. Helmke et al. observed displacement of vimentin networks, which is a kind of cytoskeletal proteins, under shear stress ${ }^{(7)}$. Recently, digital image correlation method has been applied to observe strain around cytoskeleton during cell migration ${ }^{(8)}$. The method has advantage not only in facilities of analysis but also in capability of performing high spatial resolution less than $100 \mathrm{~nm}$.

Final goal of our study is to clear mechanism of effect for mechanical loadings. As a first step of this purpose, we measured displacements on actin filaments and their binding proteins under local deformation. A set of fluorescent images of cytoskeletal proteins in fixed or live cells was processed by digital image correlation methods. We discussed deformation pattern and mechanical effect on the actin filament networks.

\section{Materials and methods}

\subsection{Cell culture}

Mouse myoblast-like cells (C2C12; RCB0987) were purchased from the RIKEN Cell Bank (Tsukuba, Japan) and cultured in low glucose Dulbecco's modified Eagle's medium (DMEM) supplemented with 10\% fetal bovine serum and 1\% antibiotics (Sigma-Aldrich, St. Louis, Missouri) in $5 \% \mathrm{CO}_{2}$ at $37{ }^{\circ} \mathrm{C}$. The cells were trypsinized and plated onto a glass dish. For live cell imaging, the culture medium in the dish was replaced with DMEM buffered with HEPES (Sigma-Aldrich).

\subsection{Construction of recombinant plasmids and transfection procedure}

A plasmid constructed to express a fusion protein of mouse $\alpha$-actinin and green fluorescent protein (GFP) is described as follows. RNA was extracted from C2C12 cells by the treatment with a phenol-based regent (ISOGEN; Nippon Gene Co., Ltd., Tokyo, Japan), followed by reverse-transcription into cDNA. Using, the cDNA, coding sequence of the mouse $\alpha$-actinin was amplified with Kod FX (Toyobo Co., Ltd., Osaka, Japan) and the following oligonucleotide primers: $\alpha$-actininF: 5'-ggctagcaccatggaccattatgattcc-3' and $\alpha$-actininR: 5'-gggaggtcgctctcgccatac-3'. PCR amplification was confirmed by analyzing the fragment on $0.8 \%$ agarose gel (Takara Bio Inc., Shiga, Japan) with standard molecular weight markers. The PCR product was digested with NheI and subcloned into the digested pAcGFP-N3 (NheI/SmaI) vector (Takara Bio Inc.). The recombinant plasmid pAcGFP- $\alpha$-actinin was amplified with E. coli strain DH5alpha (Toyobo Co., Ltd.) and was purified by cesium chloride density gradient centrifugation in order to obtain high protein expression.

In order to transfect myoblasts with the plasmid, polyethylenimine is used as a transfection reagent (kindly gifted from Dr. I. Kii). The detailed protocol is described in anywhere ${ }^{(9)}$. The maximum efficiency of expression was achieved more than $1 \sim 1.5$ day after the transfection.

\subsection{Immunofluorescence stain}

Myoblasts cultured on a glass dish were rinsed with phosphate-buffered saline (PBS, Invitrogen), followed by fixation with $4 \%$ formaldehyde/PBS for $10 \mathrm{~min}$. They were then washed twice with PBS and permeabilized with $0.5 \%$ Triton X-100/PBS for $10 \mathrm{~min}$. After blocking the nonspecific binding sites with $0.5 \%$ bovine serum albumin (BSA, Invitrogen) in PBS, the cells were stained with anti-vinculin (Sigma) in PBS by incubation for $1 \mathrm{~h}$. Subsequently, the cells were rinsed three times with PBS containing 0.5\% BSA and stained with Alexa488-phalloidin or anti-mouse IgG Alexa488 (Invitrogen) in PBS by incubation for $1 \mathrm{~h}$. To avoid loss of fluorescence intensity, the cells were covered with 
1,4-diazobicyclo[2.2.2]octane (DABCO; Wako Pure Chemicals, Osaka, Japan) diluted in glycerol.

\subsection{Impression of local deformation on cell}

Local deformation onto cells was performed via the movement of a glass needle (BF100-50-10; Sutter Instruments; Novato, CA, USA) controlled by a micromanipulator (MP-285; Sutter Instruments). Prior to the deformation, the glass needle with $100 \mu \mathrm{m}$ diameter was narrowed to be $0.1 \sim 1 \mu \mathrm{m}$ in diameter by heating and elongating (P-97; Sutter Instruments). Cells were poked by the glass needle and locally deformed by the movement of the needle with the velocity of $0.4 \mu \mathrm{m} / \mathrm{s}$. Poking depth from the cell surface to the top of the needle is not regulated in this paper, so the needle may reach at basal area of cell. The angle between glass needle and culture dish plane was fixed for all experiments and was about $30^{\circ}$. The movement of glass needle was changed 0.4 to $4 \mu \mathrm{m}$ depending on sample condition.

\subsection{Fluorescence microscopy and local displacement analysis}

Fluorescent images of cells were obtained by fluorescent microscope (TE2000-S; NIKON INSTECH Co., Ltd., Tokyo, Japan) equipped with a cooled and charge-coupled device camera (QIC-F-CLR-12-C; QIMAGING; Surrey, BC, Canada). In order to measure local displacement on a cell by the movement of the glass needle (see section 2.4.), two images of unloaded and loaded cell were applied to digital image correlation method ${ }^{(10,11)}$ or manual method. The results by the digital image correlation method were successively 3 $\times 3$ median-filtered to reduce noise from the results. In the manual method, the characteristic features on the cell were used as tracking markers, and these features in a set of unloading and loading images were manually marked by using the Image-Pro Plus (Media Cybernetics, MD, USA). Displacement vectors were analyzed by the subtraction of coordinates of a marker between the two images.

\section{Results and discussion}

We impressed cells local deformation and observed spatial changes in their cytoskeleton. Cells on a glass dish were fixed and stained with anti-vinculin, and then vinculin distribution was observed under unloading and loading (Fig. 1A). Vinculin is known to localize at the region where cells adhere to the extracellular matrices and to form insular-like structures. We also recognized such structures via the vinculin staining. To confirm that the cell was deformed locally, we magnified the area where we poked the cell with a glass needle (circle in Fig. 1A, B) and any other part in the cell (square in Fig. 1A, C). The insular-like structures at the center of the circle moved leftward (arrowhead in Fig. 1B). On the other hand, most of regions away from the deformation center did not move (square in Fig. 1C). We performed the same experiment for 4 times to confirm reproducibility. We confirmed that the cell was deformed locally.

Next, we confirmed the application of digital image correlation method to detection of intracellular displacement by comparing manual method. Cellular fluorescent images under unloading and loading (Fig. 1A, B) were processed by the two methods. Figure 2 shows a map of displacement and its direction processed by the manual method. Displacement on central areas of the deformation was about $0.4 \mu \mathrm{m}$. Degree of the displacement decreased with the distance from the center, and there was little displacement at the area over the 20 $\mu \mathrm{m}$ away from the center (outside of the brown-lined circle in Fig. 2). However, some showed valid displacement over the area (white-lined rectangular in Fig. 2). These results indicate that applied deformation propagated heterogeneously into a cell. As a whole, results of the manual method suggest that each vinculin stained structure was displaced to the direction of movement of the glass needle. The fluorescent images under unloaded and 
(A)
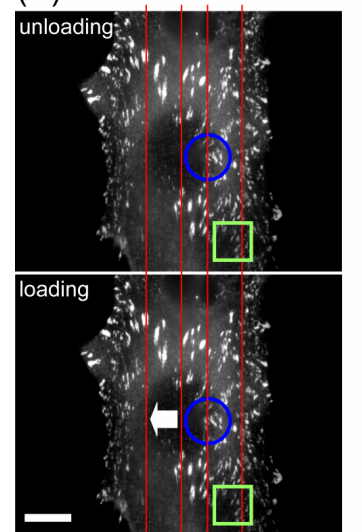

(B)

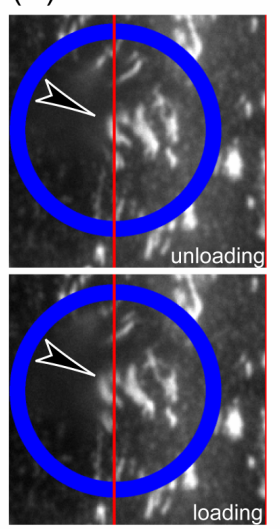

(C)

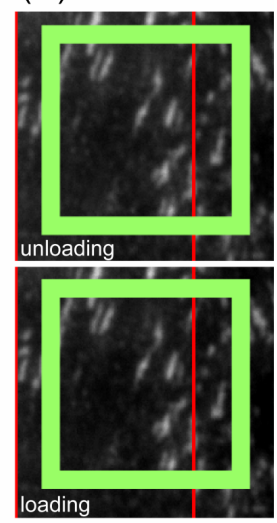

Fig. 1 Confirmation of local displacement on a myoblast cell.

A: Cells were fixed and their vinculin distribution under unloading or loading condition was observed. At the center of the circle, the cell was deformed to the direction of the arrow. The bar denotes a length of $10 \mu \mathrm{m}$. B: The areas enclosed by the circle (shown in (A)) were magnified. Arrowhead indicates a site represents movement. C: The areas enclosed by the square (shown in (A)) were magnified.

loaded were processed by the digital image correlation method (Fig. 3). Figure $3 \mathrm{~B}$ and $\mathrm{C}$ show map of displacement in $X$ (Fig. 3B) and $Y$ (Fig. 3C), where $X$ or $Y$ direction were defined in Fig. 3A. As seen in the result by manual method (Fig. 2), the cell locally deformed to the $X$ direction (break lined circle in Fig. 3B). On the other hand, there were slight displacements in the $Y$ direction (break lined circle in Fig. 3C). Map which reflects displacements on $X-Y$ plane was shown in figure 3D. The area where a degree of displacements were observed (break lined circle in Fig. 3D) corresponded to that in the $X$ displacement map (Fig. 3B). The obtained results by digital image correlation method were superior to that by manual method in the resolution. Actually, displacements were analyzed not only at the characteristic structures but around them. As the above two method showed the similar result, we confirmed that the digital image correlation method is available to detect intracellular displacement.

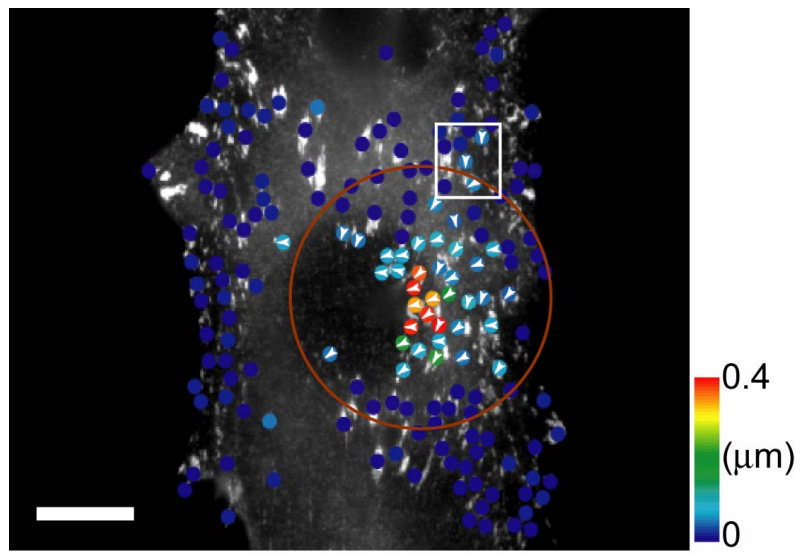

Fig. 2 Displacement map under local deformation processed by manual method.

We identified characteristic features on the cell within the two fluorescent images (unloading and loading). Displacement of each feature was analyzed by the subtraction between the coordinates of a feature. Direction of the displacement was indicated by arrowheads and degree of the displacement was expressed as a colored bar. The bar denotes a length of $10 \mu \mathrm{m}$. 
(A)

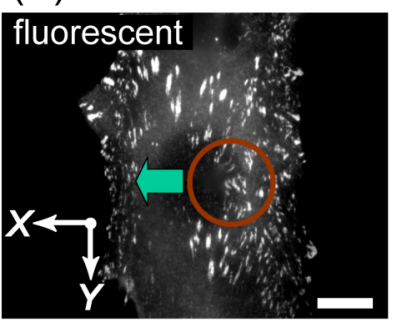

(C)

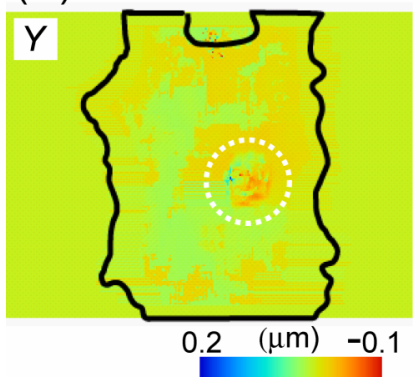

(B)

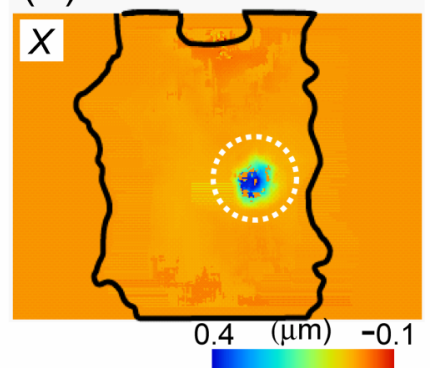

(D)

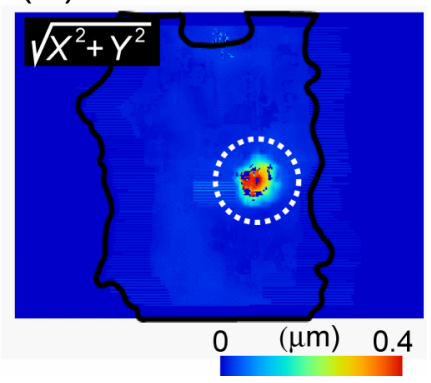

Fig. 3 Displacement map under local deformation processed by the digital image correlation method.

A: The identical fluorescent image (shown in Fig. 1A) was described again to define coordinate axes in the image. The cell was locally deformed to the direction of the green arrow. White arrows mean the $X$ or $Y$ directions, which is used in the following Fig. 3B $\sim$ D. The bar shown in the fluorescent image denotes a length of $10 \mu \mathrm{m}$. B, C, D: The figures show displacement maps in $X$ direction (B), $Y$ direction (C) or a distance map (D). The dot-lined circle means the area where the cell was locally deformed.

Heterogeneous deformation was also observed in filamentous actin stained cells. In order to observe deformation of actin networks under the loading, cells stained with Alexa 488 phalloidin were deformed via the movement of the glass needle (Fig. 4). A set of images (under unloading and loading) was processed by the digital image correlation method. Actin networks around the poked area were moved to the same direction of the glass needle movement (Fig. 4B, C). Some areas away from the deformation center also displaced (arrows in Fig. 4C, E). We performed the same experiment for 4 times to confirm reproducibility. These results suggest that actin networks were heterogeneously deformed.

We attempted to observe displacement of actin networks in live cells under external loading. The $\alpha$-actinin, which is known to be one of actin filament binding proteins, fused with GFP was exogenously expressed to live cells, and the localization was observed under loading (Fig. 5A, B). Before the loading, many fibrous structures were observed in the cell (arrows and arrowheads in Fig. 4A). The cell was locally deformed about $0.4 \mu \mathrm{m}$ to minus $X$ direction (circle in Fig. 5B). Time interval on the deformation was not more than 1 min. Fluorescent signals from the fibrous structures at the deformed region diminished (arrowheads in Fig. 4A). On the other hand, those from other sites remained bright (arrows in Fig. 5A, B). In order to observe displacement of the filamentous structures, the set of fluorescent images was processed by the digital image correlation method (Fig. 5C D). Regrettingly, significant displacement signals were not seen at the deformed region (circle in Fig. 5C, D). Some displacement signals from any other sites were seen (arrows in Fig. $5 \mathrm{C}, \mathrm{D})$. Although the poking and the deformation may influence cellular functions, we ascertained the cells to keep alive at least $1 \mathrm{~h}$ (data not shown). We performed the same experiment for 4 times to confirm reproducibility. These results imply that filamentous structures are destroyed by the application of local deformation. 
(A)

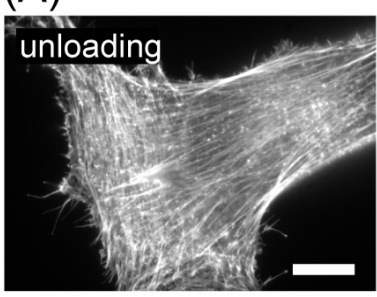

(C)

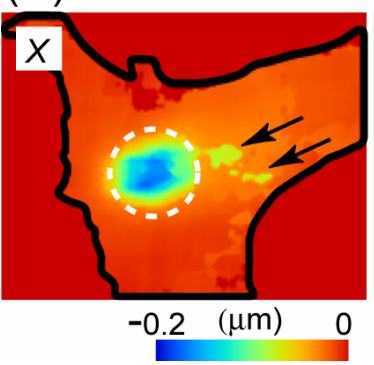

(B)

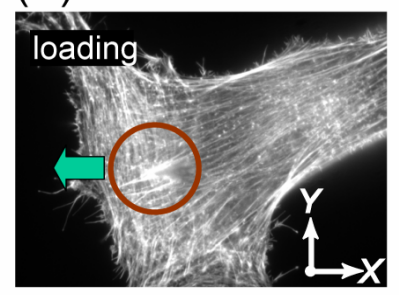

(D)

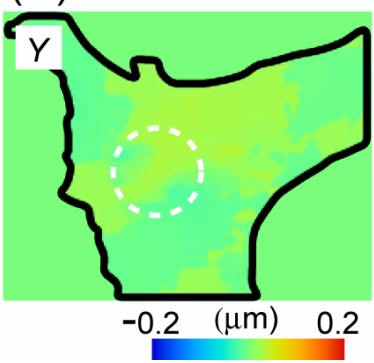

(E)

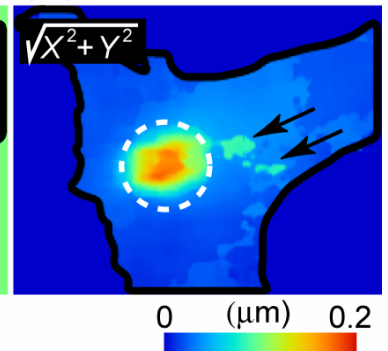

Fig. 4 Deformation of actin networks in fixed cell under loading.

A, B: Actin networks in fixed cells were deformed by movement of glass needle. The cell was poked by a glass needle at the center of the brown circle and the glass needle was moved to the direction of the green arrow (i.e. minus $X$ direction). The bar means $10 \mu \mathrm{m}$. C, D, E: The figures show displacement maps in $X$ direction (C), Y direction (D) or a distance map (E). The dot-lined circle means the area where the cell was locally deformed. Deformation discretely propagated to some areas (arrows in (C), (E)).

(A)

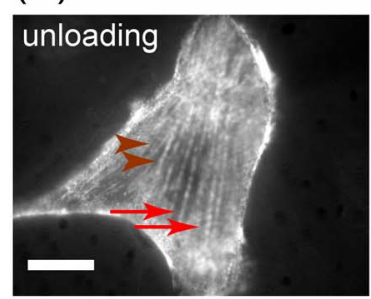

(C)

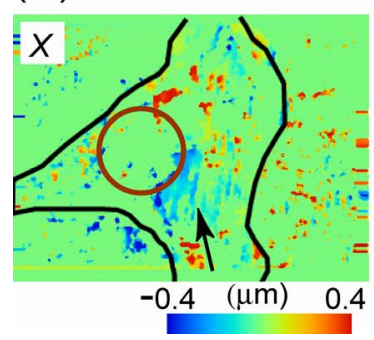

(B)

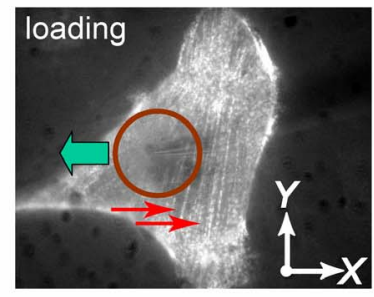

(D)

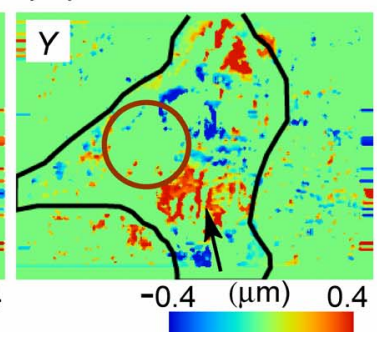

$(\mathrm{E})$

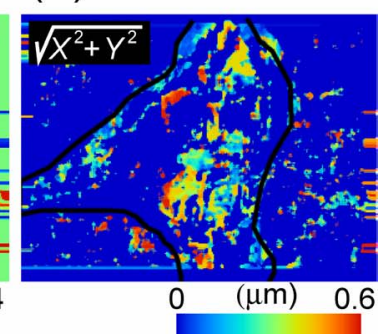

Fig. 5 Impression of local deformation on a live cell.

A, B: GFP- $\alpha$-actinin expressing cell was locally deformed at an area (circle in (B)) to the direction of green arrow (i.e. minus $X$ ). Before loading, there were some fibrous structures (arrows and arrowheads in (A)). After the loading, the fibrous structures diminished (circle in (B)). Fibrous structures away from the deformation center remained (red arrows in (B)). The bar shown in the fluorescent image denotes a length of $10 \mu \mathrm{m}$. C, D, E: The figures show displacement maps in $X$ direction (C), $Y$ direction (D) or a distance map (E). The circle means the area where the cell was locally deformed. 
It has to be pointed out that methods used here to observe intracellular displacement have an extent of limitation. Actually, intracellular structures, such as actin filament, microtubule and intermediate filaments, extend in three dimensions. However, we treat those as two dimensions to simplify measurements and analysis in this paper. From this view point, the current methods are a first approximation of a displacement mapping under mechanical loading.

Heterogeneous deformation of actin networks and focal adhesions may result from differences of mechanical strength between the structures, and/or may involve other mechanical transmitters. Cell-substrate adhesion force is proposed to depend on degree of their maturation ${ }^{(12)}$, and also cellular stiffness map mainly reflecting force on actin fiber networks is reported to be heterogeneous ${ }^{(13)}$. In the case, mechanically weak structures will tend to displace widely than strong structures do. This is a likely reason for the heterogeneous deformation. Other reason is that other mechanical structures connect focal adhesions and actin filaments, and the mechanical structures dislocate focal adhesions and actin filaments not only at a little distance but also at a little separated place without affecting focal adhesions and actin filaments with which mechanical structures don't interact. Microtubule is known to interact with actin filaments ${ }^{(14)}$. Taken together, other cytoskeletal may be candidate for the heterogeneous deformation.

Local deformation may cause disruption of actin filaments by induction of biochemical reactions. The same degree of deformation was applied to fixed cells and live cells, however, in the case of live cell, fluorescent signals decreased locally without decreasing at other regions (Fig. 5A, B). The decrease means the disruption of actin filament by the induction of local deformation because fibrous structures at other regions remained bright. One of possible explanations for the disruption is activation of biochemical signal cascades. In the case of fixed cell, biochemical reactions must be restricted. On the other hand, biochemical signals are allowed to occur in live cells. A biochemical signal may respond to deformation on actin filaments and disrupt them, however, there is no evidence to support the idea. Further experiments are required to elucitate the problem.

\section{Conclusion}

We estimated the intracellular displacement under local deformation by the comparison of manual method and digital image correlation method. We confirmed that the deformation adequately applied to cells. The deformation pattern is not always uniform but heterogeneous. Some actin filaments were disrupted by the deformation in live cells.

\section{Acknowledgements}

This work was supported in part by the Collaborative Research Program of Research Institute for Applied Mechanics, Kyushu University and by the CASIO Science Promotion Foundation.

\section{References}

(1) Blain, E. J., Involvement of the cytoskeletal elements in articular cartilage homeostasis and pathology, International Journal of Experimental Pathology, Vol. 90 (2009), pp. 1-15.

(2) Grossi, A., Yadav, K. and Lawson, M. A., Mechanical stimulation increases proliferation, differentiation and protein expression in culture: stimulation effects are substrate dependent, Journal of Biomechanics, Vol. 40 (2007), pp. 3354-3362.

(3) Li, Z., Kupcsik, L., Yao, S. J., Alini, M. and Stoddart, M. J., Mechanical Load Modulates Chondrogenesis of Human Mesenchymal Stem Cells through the TGF- $\beta$ Pathway, Journal of 
Cellular and Molecular Medicine, (2009), in printing.

(4) Tzima, E., del Pozo, M. A., Shattil, S. J., Chien, S. and Schwartz, M. A., Activation of integrins in endothelial cells by fluid shear stress mediates Rho-dependent cytoskeletal alignment, The EMBO Journal, Vol. 20 (2001), pp. 4639-4647.

(5) Costa, K. D., Hucker, W. J. and Yin, F. C., Buckling of actin stress fibers: a new wrinkle in the cytoskeletal tapestry, Cell Motility and the Cytoskeleton, Vol. 52 (2002), pp. 266-274.

(6) Hu, S. H., Chen, J.X., Fabry, B., Numaguchi, Y., Gouldstone, A., Ingber, D. E., Fredberg, J. J., Butler, J. P., and Wang, N., Intracellular stress tomography reveals stress focusing and structural anisotropy in cytoskeleton of living cells, American Journal of Physiology-Cell Physiology, Vol. 285 (2003), pp. C1082-C1090.

(7) Helmke, B.P., A.B. Rosen, and P.F. Davies., Mapping mechanical strain of an endogenous cytoskeletal network in living endothelial cells, Biophysical Journal, Vol. 84 (2003), pp. 2691-2699.

(8) Adachi, T., Okeyo, K. O., Shitagawa and Y., Hojo, M., Strain field in actin filament network in lamellipodia of migrating cells: implication for network reorganization, Journal of Biomechanics, Vol. 42 (2009), pp. 297-302.

(9) Chenuet, S., Martinet, D., Besuchet-Schmutz, N., Wicht, M., Jaccard, N., Bon, A. C., Derouazi, M., Hacker, D. L., Beckmann, J. S. and Wurm, F. M., Calcium Phosphate Transfection Generates Mammalian Recombinant Cell Lines With Higher Specific Productivity Than Polyfection, Biotechnology and Bioengineering, Vol. 101 (2008), pp. 937-945.

(10) Umezaki, E. and Futase, K., Deformation behaviour of the cross-section of a heat-sealed area in plastic film used for liquid package bags, Journal of Strain Analysis for Engineering Design, Vol. 32 (1997), pp. 29-35.

(11) Cao, Y. F., Bly, R., Moore, W., Gao, Z., Cuitino, A. M. and W. Soboyejo., On the measurement of human osteosarcoma cell elastic modulus using shear assay experiments, Journal of Materials Science-Materials in Medicine, Vol. 18 (2007), pp. 103-109.

(12) Möhl, C., Kirchgessner, N., Schäfer, C., Küpper, K., Born, S., Diez, G., Goldmann, W. H., Merkel, R. and Hoffmann, B., Becoming stable and strong: the interplay between vinculin exchange dynamics and adhesion strength during adhesion site maturation, Cell Motility and the Cytoskeleton, Vol. 66 (2009), pp. 350-364.

(13) Haga, H., Sasaki, S., Kawabata, K., Ito, E., Ushiki, T. and Sambongi, T,. Elasticity mapping of living fibroblasts by AFM and immunofluorescence observation of the cytoskeleton, Ultramicroscopy, Vol. 82 (2000), pp. 253-258.

(14) Kalil, K. and Dent, E. W., Touch and go: guidance cues signal to the growth cone cytoskeleton, Current Opinion in Neurobiology, Vol. 15 (2005), pp. 521-526. 